\title{
Sanción de conductas anticompetitivas extraterritoriales en Estados Unidos y la Unión Europea
}

\author{
Jaime Arancibia Mattar*
}

\section{RESUMEN}

Este trabajo aborda el poder de sanción de conductas anticompetitivas extranjeras que afectan la libre competencia en los mercados internos de Estados Unidos y la Unión Europea. Destaca el ejercicio de competencia doméstica basado en la territorialidad de los efectos adversos de la conducta, que deben ser sustanciales y directos. Asimismo, identifica algunos criterios de deferencia o cortesía que procuran conciliar los intereses jurisdiccionales de los países afectados por ilicitos transfronterizos.

Libre competencia - jurisdicción - extraterritorial

\section{Antitrust sanctions on extraterritorial conducts in the United States and the European Union}

\begin{abstract}
This article discusses the United States and European Union's sanctioning powers over extraterritorial antitrust infringements that affect their internal markets. It remarks the domestic sanction of those actions on the ground that they have produced an adverse impact on the domestic territory, which must be substantial and direct. In addition, it identifies some deferential or comity criteria which contributes to conciliate jurisdictional interests affected by cross border infringements.
\end{abstract}

Antitrust - jurisdiction - extraterritorial

* Licenciado en Derecho, Pontificia Universidad Católica de Chile. Doctor en Derecho, Universidad de Cambridge, Inglaterra. Profesor de Derecho Administrativo y Constitucional, Universidad de los Andes, Chile. Correo electrónico: jarancibia@uandes.cl.

Artículo recibido el 8.3.2019 y aceptado para su publicación el 19.5.2020. 


\section{INTRODUCCIÓN ${ }^{1}$}

E ste trabajo tiene por objeto la identificación de los criterios que rigen el juzgamiento y sanción de conductas extraterritoriales que producen efectos anticompetitivos en Estados Unidos y la Unión Europea. La sistematización de este corpus dogmático se enmarca en una línea de investigación que pretende proponer, en un estudio ulterior, algunas orientaciones para la sanción en Chile de ilícitos de libre competencia fraguados en el extranjero, materia escasamente tratada por la doctrina nacional ${ }^{2}$.

A raíz del fenómeno de globalización comercial o tendencia de los mercados a sobrepasar los límites geográficos de los Estados, los atentados a la libre competencia pueden provenir de hechos, actos o convenciones ejecutados o celebrados en países extranjeros, distintos del lugar o lugares donde pretenden ser juzgados. Un cartel internacional, una fusión o toma de control de empresas internacionales por sobre los umbrales permitidos a nivel doméstico, o un abuso de posición dominante pueden afectar seriamente la libertad de competir en suelo nacional. Es por esta razón que los ordenamientos de libre competencia en países industrializados han elaborado un acervo de criterios jurisdiccionales respecto de estas conductas, que procura compatibilizar los intereses soberanos de los países con la necesidad de hacer frente a ilícitos transnacionales favorecidos por la alta comunicabilidad comercial. En este sentido, el juzgamiento de conductas extranjeras plantea desafíos importantes a instituciones clásicas del derecho procesal como el juez natural territorial ${ }^{3}$, las medidas precautorias ${ }^{4}$, la prueba y la ejecución de sentencias ${ }^{5}$, entre otras.

Debido a que estos criterios son aplicados actualmente por dos de los principales aliados comerciales de Chile $^{6}$, es preciso identificarlos y analizarlos en una labor que precede a una propuesta de bases para el juzgamiento en nuestro país de conductas extranjeras -al menos de agentes económicos con sede en Estados Unidos y Europa, por razones de reciprocidad- que produzcan efectos anticompetitivos en suelo nacional.

Para efectos de lo anterior, este trabajo considera el estudio de los siguientes principios y criterios: a) criterios vigentes de competencia del órgano doméstico para sancionar conductas foráneas en razón de un factor de conexión relevante, $v$. gr. efectos anticompetitivos; b) criterios de cortesía (comity) o respeto por la jurisdicción sancionadora

\footnotetext{
${ }^{1}$ Este artículo fue escrito en el marco del Proyecto Fondecyt Regular $\mathrm{N}^{\circ} \mathrm{N}^{\mathrm{o}} 1161741$, acerca de "Análisis y revisión dogmática del derecho administrativo sancionador en Chile a partir de su parte especial”, en el que el autor es coinvestigador. Todas las traducciones de textos en inglés son nuestras.

${ }^{2}$ Podríamos mencionar como excepción el trabajo de Antonio Bascuñán en materia de colusión, aunque utilizando un enfoque más bien penal que no estimamos del todo aplicable a infracciones administrativas o civiles en libre competencia. Bascuñán Rodríguez, Antonio, Estudios sobre Colusión, Thomson Reuters, 2016, Capítulo I, pp. 7 y ss.

3 Ormazábal Sánchez, Guillermo, 2016, pp. 117 a 152.

${ }^{4}$ Gilsanz Usunaga, Javier, 2010, pp. 311 a 371.

${ }^{5}$ Gascón InChausti, Fernando, 2017.

${ }^{6}$ Cfr. Ministerio de Relaciones Exteriores, 2018.
} 
extranjera; c) limitación del poder sancionador doméstico debido al principio non bis in idem en relación con la jurisdicción extranjera.

\section{El JUZGamiento de CONDUCTAS FORÁNEAS SEGÚN UN FACTOR DE CONEXIÓN RELEVANTE}

Luego de un período inicial de declaraciones de incompetencia basada en una deferencia absoluta a la jurisdicción soberana de países extranjeros ${ }^{7}$, la jurisprudencia norteamericana y comunitaria europea evolucionó hacia una dogmática actual que extiende la competencia territorial a cualquier ilícito perjudicial para el interés nacional, aunque sus elementos estén conectados con un territorio extranjero. Estos ilícitos, por tanto, requieren un mínimo de conexión razonable con el territorio de asiento del órgano que conoce $(v \cdot g r$. nacionalidad del autor o de la víctima, ejecución de un elemento de la infracción, afectación de intereses públicos relevantes o comunes a la humanidad).

El factor de conexión más relevante de esta posición es el efecto ilícito de la conducta en el territorio jurisdiccional. De ahí su denominación como doctrina de los "efectos" o "delitos a distancia", amparada por el precedente de la Corte Permanente de Justicia Internacional en el caso Lotus $^{8}$.

Un primer indicio de jurisdicción extraterritorial en el derecho de libre competencia estadounidense se aprecia en la "Ley para proteger el intercambio y el comercio frente a restricciones y monopolios ilícitos" ${ }^{\text {, }}$, conocida como la Ley Sherman en honor al senador que la propuso. Esta normativa, aprobada en 1890, sanciona a la persona o personas que restrinjan el intercambio o el comercio entre los estados de la unión, "o con naciones extranjeras"10. Además, la sección octava de la ley establece que: "la palabra persona, o personas, utilizada en esta ley se entiende que incluye a las corporaciones o asociaciones que existen bajo o autorizadas por las leyes de Estados Unidos, de cualquiera de los territorios, de cualquier Estado, o la leyes de cualquier país extranjero".

Sin embargo, como vimos, la jurisprudencia inicial interpretó de modo restrictivo estas disposiciones, procurando extender la competencia de los tribunales únicamente a ilícitos cometidos en el territorio de Estados Unidos. Posteriormente, los jueces evolucionaron hacia un régimen de juzgamiento de actos extraterritoriales, cometidos por empresas nacionales o extranjeras, que produjeran efectos anticompetitivos en el territorio de Estados Unidos.

${ }^{7}$ Corte Suprema de Estados Unidos, 26.4.1909, No 686. Una reacción similar se aprecia en las Cortes británicas. Por ejemplo, Cámara de los Lores del Reino Unido, 1.12.1977, Rol No 235 ( $\mathrm{N}^{\circ} 1$ y $\mathrm{N}^{\circ} 2$ ). Cfr. SAmie, 1981, p. 58; Blythe, 1983, p. 99.

${ }^{8}$ Corte Permanente de Justicia Internacional, 7.9.1927, Rol A-No 10.

9 The Sherman Antitrust Law, 1890.

${ }^{10}$ Esta expresión es utilizada en cada una de las secciones en que se describe la conducta típica: $1^{\circ}$, $2^{\circ}, 3^{\circ}$ y $6^{\circ}$. 
El primer precedente en esta materia es el caso Aluminium ${ }^{11}$, donde la Corte de Apelaciones del Segundo Circuito ejerció jurisdicción sobre una empresa con domicilio en Canadá por celebrar acuerdos de producción de aluminio para suprimir o restringir la importación del producto en Estados Unidos. La sentencia aplicó como derecho vigente y reconocido por los estados el "que cualquier Estado puede imponer responsabilidades, incluso a personas no sometidas a su jurisdicción, por conductas cometidas más allá de sus fronteras que tengan consecuencias en el territorio contrarias al derecho interno”.

Esta mención expresa a empresas foráneas es especialmente relevante para el tema en estudio. La responsabilidad por conductas extraterritoriales que produjeran efectos en Estados Unidos se extendería incluso a personas naturales o jurídicas con nacionalidad y domicilio extranjero. Esta doctrina fue confirmada por la Corte del Noveno Circuito en Timberlane ${ }^{12}$, sobre atentados al mercado de la madera en Estados Unidos mediante actividades en Honduras: "La ley (Sherman) podría cubrir actividades en el exterior de ciudadanos extranjeros y americanos".

Un primer ejemplo concreto de aplicación de la ley a una empresa extranjera es Daishowa $^{13}$, en que la Corte del Distrito Norte de California juzgó a importadores japoneses de chips de madera acusados de formar un cartel comprador, dividir a los proveedores americanos, fijar precios y boicotear las actividades de un exportador, causando de este modo una caída artificial del precio de mercado.

Otro ejemplo viene dado por el caso Hartford ${ }^{14}$, en que compañías reaseguradoras londinenses fueron demandadas en Estados Unidos por actos de conspiración y boicot para forzar cambios en las pólizas de los seguros en dicho país. Al respecto, la Corte Suprema señaló que "está bien establecido ahora que la Ley Sherman se aplica a una conducta foránea que tuvo por objeto producir y que, de hecho, produjo un efecto sustancial en los Estados Unidos" 15 .

Si bien la jurisprudencia y la legislación de Estados Unidos han sido pioneras en la aplicación de la doctrina de los efectos en libre competencia ${ }^{16}$, es preciso señalar que luego le sigue la jurisprudencia de la Unión Europea ${ }^{17}$, que ha aplicado criterios similares aunque con menor grado de sofisticación según veremos. Básicamente, los aportes de esta última se han concentrado en la legitimación pasiva común de matrices y filiales ubicadas en distintos países, sobre la base de la doctrina de la unidad económica singular, y en un desarrollo de la doctrina de los efectos similar a la de Estados Unidos.

${ }^{11}$ Corte de Apelaciones de Estados Unidos para el Segundo Circuito, 12.3.1945, Rol No 144.

12 Corte de Apelaciones de Estados Unidos para el Noveno Circuito, 27.12.1976, Rol N ${ }^{\text {os. } 74-2142,}$ 74-2354, 74-2812 and 74-2813.

${ }^{13}$ Corte del Distrito Norte de California, 1982, Rol No 1982-2 Trade Cas. (CCH) P64,774.

${ }^{14}$ Corte Suprema de Estados Unidos, 28.6.1993, Rol No 91-1111. Véase DAM, 1993, 1994, p. 289; Kramer, 1995, p. 750; Gupta, 1996, p. 2287.

${ }^{15}$ Ibíd., 796. Ver también Matsushita Elec. Industrial Co. v. Zenith Radio, Corte Suprema de Estados Unidos, 26.3.1986, Rol No 83-2004.

16 ONGMAN, $1977,733$.

17 Torremans, 1996, p. 280. 


\section{Requisitos de juzgamiento}

En relación con los requisitos para ejercer competencia, el fallo de Aluminium precisó que la Ley Sherman sanciona conductas de colusión realizadas en el extranjero que cumplan un doble requisito: a) tener por objeto afectar las importaciones o exportaciones de Estados Unidos; y b) producir un efecto real y verificable respecto de ellas ${ }^{18}$.

Es importante señalar que estos criterios de competencia judicial fueron posteriormente reconocidos por la Ley Sherman. En efecto, la reforma por el perfeccionamiento de la libre competencia en el comercio exterior ${ }^{19}$ añadió un acápite $\$ 7$ a la ley para extender su aplicación a conductas anticompetitivas que involucren intercambio o comercio internacional. La pretensión de juzgamiento de conductas foráneas es evidente en la historia y texto de la normativa.

En primer lugar, conviene considerar el cambio de redacción de la ley durante su tramitación. El propósito original de la normativa era promover el comercio exterior de Estados Unidos eximiendo a sus exportadores de la Ley Sherman, de forma que pudieran realizar prácticas anticompetitivas impunes en la medida que no afectaran el comercio interno. Es por esta razón que el texto primario del precepto se refería al "intercambio y comercio de exportación”. Sin embargo, la palabra exportación fue posteriormente eliminada y reemplazada por una de alcance general "(distinto al de importación)". Según se explica en la historia de la ley ${ }^{20}$, esta modificación tenía por objeto eximir también de la Ley Sherman a las conductas foráneas inocuas al comercio de Estados Unidos. Luego, a contrario sensu, sí estarían sujetas aquellas perniciosas para el mismo.

Específicamente, la Sección 6a de la ley reconoce expresamente jurisdicción sobre conductas extranjeras, supeditada al cumplimiento de los siguientes requisitos ${ }^{21}$ :

“1) Esa conducta tenga un efecto directo, sustancial y razonablemente previsible

(A) sobre el intercambio o el comercio que no es intercambio o comercio con naciones extranjeras, o sobre el intercambio de importación o comercio de importación con naciones extranjeras; o

18 Acerca del efecto real en el comercio de Estados Unidos, véase también Power East v. Transamerica Delaval, Inc., Corte de Distrito de Nueva York S.D., 21.1.1983, Rol No 82 Civ. 4269 (KTD); United States v. Westinghouse Elec. Corp., Corte de Distrito de California N.D., 20.10.1978, Rol C-70-852 SAW.

19 The Foreign Trade Antitrust Improvements Act, 1982.

${ }^{20}$ Congreso de Estados unidos, 1982, p. 2487, 2487-2488, 2494-2495.

21 (1) such conduct has a direct, substantial, and reasonably foreseeable effect

(A) on trade or commerce which is not trade or commerce with foreign nations, or on import trade or import commerce with foreign nations; or

(B) on export trade or export commerce with foreign nations, of a person engaged in such trade or commerce in the United States; and

(2) such effect gives rise to a claim under the provisions of sections 1 to 7 of this title, other than this section. 
(B) sobre el intercambio de exportación o comercio de exportación con naciones extranjeras, de una persona comprometida en ese intercambio o comercio en los Estados Unidos; y

2) Tal efecto da lugar a una reclamación conforme a las disposiciones de las secciones 1 a 7 de este título, distintas a las de esta sección”.

Es interesante notar que este test para la sanción de conductas foráneas, que requiere un efecto "sustancial" en el comercio, es más exigente que el relativo a conductas internas, que solo reclama un efecto en el comercio entre estados ${ }^{22}$. Por esta razón, un demandante podría verse tentado a calificar el conflicto simplemente de "doméstico" para evitar la prueba de dicho efecto. Un ilícito propicio para tal opción sería aquel en que participan empresas de Estados Unidos y extranjeras.

No obstante esta posibilidad, la jurisprudencia ha fijado criterios para identificar efectos no sustanciales. Es el caso de Montreal Trading ${ }^{23}$, en que se demandó la negativa de filiales canadienses a vender potasa producida en Canadá a otra empresa canadiense con intenciones de revenderla en Corea del Norte. La Corte del Décimo Circuito señaló que "cuando los contactos con Estados Unidos son pocos, los efectos sobre el comercio americano mínimos, y los elementos extranjeros abrumadores, nosotros no aceptamos jurisdicción”.

A mayor abundamiento, la interpretación de los presupuestos de competencia que exige la Foreign Trade Antitrust Improvements Act of 1982 (FTAIA) ${ }^{24}$ estadounidense ha sido fuente de controversias que han permitido a la doctrina de los efectos un avance dogmático relevante. Este se aprecia principalmente respecto de qué se entiende por “conducta foránea" y "efecto directo".

\section{Conducta foránea}

En relación con el carácter foráneo de la conducta, el problema se plantea principalmente en ilícitos con participación de agentes locales y extranjeros.

Las prácticas de empresas estadounidenses realizadas en el extranjero con el propósito de afectar el comercio de su país están especialmente sujetas a la Ley Sherman, debido a la conjunción de dos factores de competencia: la doctrina de los efectos y la nacionalidad del demandado. Por esta razón, en casos de cartel o colusión con compañías extranjeras, Estados Unidos y sus tribunales han preferido litigar exclusivamente con la empresa nacional.

Así por ejemplo, en Timken ${ }^{25}$, la Corte Suprema juzgó actos de colusión y conspiración cometidos por una empresa de rodamientos norteamericana con una sociedad

\footnotetext{
${ }^{22}$ Véase McLain v. Real Estate Bd. of New Orleans, Corte Suprema de Estados Unidos, 8.1.1980, Rol $\mathrm{N}^{\mathrm{o}} 78-1501$.

${ }^{23}$ Corte de Apelaciones de Estados Unidos para el Décimo Circuito, 14.10.1981, Rol No 79-1999.

${ }^{24}$ The Foreign Trade Antitrust Improvement Act. 1982.

${ }^{25}$ Corte Suprema de Estados Unidos, 4.6.1951, Rol No 352.
} 
británica y otra francesa en las que tenía participación. Estos tenían por objeto dividir territorios, fijar precios, eliminar competidores y participar en un cartel para restringir las importaciones y exportaciones en Estados Unidos.

Es digno de notar que la compañía fue sancionada con la orden de enajenar todas sus acciones en las empresas extranjeras, debido a que habían sido adquiridas como parte de un plan para promover restricciones comerciales ilegales. Sin embargo, esta medida fue objeto de un voto disidente por algunos jueces de la Corte, por estimarla desproporcionada y ajena al fin para el que fue creada, esto es, la restauración de la competencia a futuro y no el castigo por conductas pasadas.

A su vez, en Dee-K Enterprises ${ }^{26}$, un caso de conspiración de productores asiáticos y compañías norteamericanas para fijar el precio de los elásticos de goma en Estados Unidos, la demandante alegó que la conducta no era "totalmente foránea” porque participaron empresas locales y se concretó en una venta directa del producto al interior del país. En consecuencia, se trataba de un ilícito "doméstico" que no exigía acreditar un efecto "sustancial" en el comercio.

Sin embargo, la Corte de Apelaciones del Cuarto Distrito precisó que la mera participación de empresas locales en el ilícito no basta para calificar un conflicto de "doméstico". Es necesario acreditar, además, que la conducta tuvo lugar "primariamente" en tierras americanas. Al respecto, citó como ejemplo el caso Carpet Group ${ }^{27}$, en que una Corte calificó de "local" una conspiración de entidades estadounidenses y extranjeras para proteger a los distribuidores locales de alfombras orientales importadas, en atención a que la mayoría de los conspiradores y varios objetivos de su presión estaban en Estados Unidos, y a que numerosas reuniones tuvieron lugar en suelo americano. En cambio, continuó la Corte, esta preeminencia de factores locales no se dio en el caso In re Uranium Antitrust Litig 28 , en que el tribunal calificó de "foránea" una conspiración de veinte compañías nacionales y nueve extranjeras que se reunieron en seis países para fijar el precio del uranio en el mercado mundial, principalmente porque el mercado objeto de la conducta era global.

Aplicando la misma lógica, la Corte descartó el criterio que califica como "foránea" per se cualquier conducta acordada en reuniones en el extranjero. Semejante razonamiento desconocería otros elementos como la nacionalidad de los participantes, la ubicación y ámbito de su mercado, y el lugar de los actos distintos a los acuerdos conspirativos. Su aplicación estricta podría llevar, por ejemplo, a calificar de "extranjera" una conspiración de productores locales que se reunieron fuera del país solo para fijar el precio; o a calificar de "doméstica" una conspiración de empresas en su propio país para fijar precios en un mercado extranjero que no afecta el comercio interno, hipótesis no sujeta a la jurisdicción americana ${ }^{29}$. Por estas razones, concluyó el fallo, "el universo de factores

${ }^{26}$ Corte de Apelaciones de Estados Unidos para el Cuarto Distrito, 30.7.2002, Rol No 01-1894.
${ }^{27}$ Corte de Apelaciones de Estados Unidos para el Tercer Circuito, 8.9.2000, Rol No 99-5931.
${ }^{28}$ Corte de Apelaciones de Estados Unidos para el Séptimo Circuito, 15.2.1980, Rol no citado.
${ }^{29}$ De hecho, ésta fue la hipótesis del caso Kruman v. Christie's International Plc., Corte de Distrito de Nueva York S.D., 29.1.2001, Rol No 00Civ.6322(LAK), sobre servicios de remate, en que la sentencia sostuvo que no existía jurisdicción para conocer de la conducta. 
relevantes para nuestro análisis jurisdiccional debe ser mayor que el del lugar específico de los actos conspirativos".

Finalmente, conforme con estas distinciones, la Corte en Dee-K Enterprises calificó la conducta como primariamente "foránea", sujeta a la exigencia de efecto "sustancial", porque el objetivo de la conspiración era el mercado global, todas las reuniones se realizaron en el Sudeste Asiático, y participaron en ellas solo dos personas con oficinas en compañías americanas filiales de las asiáticas.

Adicionalmente, la sentencia desestimó que la venta directa del producto a los consumidores determina, por sí solo, el carácter doméstico de la conducta. No solo desconocería otros factores como la nacionalidad de los conspiradores, el lugar de las reuniones y la naturaleza del mercado objetivo. También significaría extender la jurisdicción de Estados Unidos a una variedad de situaciones no relacionadas directamente con el país. Por ejemplo, casos en que el demandante no ha sido comprador ni vendedor en el mercado nacional, o incluso conductas foráneas que no producen daño al comercio pero que la legislación castiga como ilícitos per se.

Por ejemplo, en Zenith ${ }^{30}$, una empresa fabricante de aparatos de radio y televisión interpuso una demanda reconvencional en contra de la compañía titular de las patentes (HRI) y su matriz en Estados Unidos (Hazeltine Corporation), por conspirar con empresas extranjeras para rechazar todas sus solicitudes de licencia y distribución en el extranjero. La demanda fue interpuesta únicamente en contra de la empresa filial y no la matriz, pues las partes estipularon en el juicio principal que "para los efectos de esta litigación, el demandante y su pariente Hazeltine serán consideradas como una sola y la misma compañía”.

Sin embargo, la Corte Suprema sostuvo que, a pesar de dicha cláusula, la sentencia de fondo no producía efectos respecto de la sociedad matriz que no compareció en el juicio. Dicha estipulación "no era un sustituto adecuado de los métodos normales para obtener jurisdicción sobre una persona o corporación” y fue suscrita por un empleado de la empresa sin poderes de representación. Además, señaló la Corte, el hecho de que la sociedad filial y la matriz fuesen alter ego no formó parte de la litis.

En materia de legitimación pasiva, es admisible destacar la sentencia de la Corte Europea de Justicia (CEJ) en el caso Imperial Chemical ${ }^{31}$, respecto de colusión de empresas extranjeras productoras de colorante para afectar, inter alia, los precios y competencia en el mercado europeo por intermedio de sus filiales. En vez de recurrir a la doctrina de los efectos de la actuación de la matriz extranjera, la Corte consideró dicha conducta como doméstica en razón de que la matriz y la filial conformaban una entidad

\footnotetext{
${ }^{30}$ Corte Suprema de Estados Unidos, 19.5.1969, Rol No 49.

${ }^{31}$ CEJ, 14.7.1972, Rol Caso 48/69.
} 
económica única ${ }^{32}$. Esta doctrina fue aplicada también en los casos Europemballage E $^{33}$ Istituto Chemioterapico ${ }^{34}$.

De lo anterior se desprende, entonces, que el carácter "doméstico" o "foráneo" de una conducta en la que participan sujetos nacionales y extranjeros dependerá de la prevalencia del conjunto de factores territoriales o extraterritoriales, entre los que destacan la nacionalidad de los participantes, sus actividades, mercados objetivos y efectos, así como la existencia de empresas relacionadas en el territorio jurisdiccional.

\section{Efecto directo}

Según la legislación de Estados Unidos, una conducta anticompetitiva foránea solo puede ser juzgada en el país si produce, entre otros, un efecto "directo" en el comercio de dicho país. La doctrina ${ }^{35}$ y la jurisprudencia han procurado dar una interpretación precisa a esta exigencia.

Por ejemplo, la sentencia en el caso LSL Biotechnologies ${ }^{36}$, sobre elaboración de semillas transgénicas, definió efecto "directo" en los mismos términos que la Corte Suprema al aplicar la ley de inmunidad de soberanía extranjera ${ }^{37}$ : "el que se sigue como consecuencia inmediata de la actividad del demandado" 38 . Por tanto, continuó el fallo, el acuerdo entre un competidor nacional y otro extranjero para restringir una supuesta e incierta capacidad de innovación del segundo gozaría de inmunidad de jurisdicción en Estados Unidos, pues no produciría efectos directos en su comercio.

Así también, se ha señalado que la reducción del flujo de divisas enviadas hacia una sociedad matriz estadounidense por parte de filiales extranjeras afectadas por conductas anticompetitivas no constituye efecto directo para fines jurisdiccionales. En Intel ${ }^{39}$, un fabricante norteamericano de microprocesadores demandó a un competidor local por celebrar acuerdos de distribución exclusiva con proveedores extranjeros que afectaron la venta de sus filiales y, consecuencialmente, el envío de fondos hacia la matriz en Estados Unidos. El demandante alegó que esta reducción de ingresos afectaba su capacidad para ofrecer descuentos a los consumidores locales y que, por tanto, se configuraba un efecto adverso directo para el comercio de Estados Unidos. Sin embargo, la Corte del Distrito de Delaware rechazó la demanda por considerar que la cadena de consecuencias de la

${ }^{32}$ Es importante precisar que la doctrina europea de la unidad económica de la empresa matriz con sus filiales o coligadas para efectos de ilícitos anticompetitivos, en la medida que se cumplan los requisitos de participación correspondientes, sirve no solo para efectos extraterritoriales sino también domésticos. $C f r$. Signes de Mesa, Fernández, y Fuentes, 2013, p. 79; Odudu, Bailey, 2014, pp. 1721-1757.

33 CEJ, 21.2.1973, Rol Caso C- 6/72.

${ }^{34}$ CEJ, 6.3.1974, Rol casos acumulados 6 and $7 / 73$.

35 Beckler, y KirTland, 2003, p. 11.

${ }^{36}$ Corte de Apelaciones de Estados Unidos para el Noveno Circuito, 11.8.2004, Rol No 02-16472.

${ }^{37}$ Foreign Sovereign Immunities Act 1976, modificada en 1997.

${ }^{38}$ Republic of Argentina v. Weltover, Inc., Corte Suprema de Estados Unidos, 12.6.1992, Rol No 91-763.

39 Corte de Distrito de Delaware, 26.9.2006, Rol MDL 05-17174JF, CIV.A.05-441-JJF. Cfr. Prete, 2018, pp. 1-9. 
conducta extranjera estaba tan colmada de "giros y vueltas" que solo producía efectos indirectos o de "onda expansiva" (ripple effect) en el comercio doméstico.

Otro ejemplo de afectación no inmediata o indirecta es aquella producida en mercados conexos a aquel en que incide la conducta acusada. Así, por ejemplo, en el caso Motorola ${ }^{40}$, la Corte del Séptimo Circuito declaró que la colusión que afectó al mercado de las pantallas de cristal líquido en el extranjero no produjo un efecto directo en el mercado de los teléfonos móviles en Estados Unidos. Por tanto, no sería de competencia de jueces nacionales.

Sin embargo, jurisprudencia más reciente ha sostenido una noción de efecto directo menos estricta y más cercana a las pretensiones del Departamento de Justicia de Estados Unidos $^{41}$. Así, por ejemplo, en el caso Minn-Chem ${ }^{42}$, sobre colusión internacional en el mercado de la potasa, la Corte del Séptimo Distrito sostuvo que el requisito de efecto directo se refiere a un nexo causal razonablemente próximo, de modo que solo excluye actividades que sean "demasiado remotas" respecto de sus efectos en el comercio estadounidense. Este criterio deja abierta la posibilidad de sancionar incluso conductas que no se dirigen directamente a afectar el mercado concernido. En efecto, en el caso concreto, la conducta acusada consistía en elevar el precio de la potasa en mercados distintos al estadounidense. Empero, a juicio de la Corte, la conducta acusada produjo igualmente un efecto directo porque Estados Unidos es uno de los principales consumidores mundiales de potasa, de modo que una colusión que logró elevar el precio del insumo en otros países provocó un sobreprecio inmediato en su jurisdicción.

Una interpretación similar siguió la Corte del Segundo Circuito en el caso Lotes ${ }^{43}$. Una empresa estadounidense, fabricante de USB (Universal Serial Bus), demandó a una empresa China por haber iniciado litigios de patentes en su contra ante las Cortes de dicho país. Alegó que estos juicios tendrían el efecto de aumentar injustamente el valor de sus productos en Estados Unidos, afectando así la libre competencia. La Corte criticó el criterio consignado en el citado caso LSL Biotechnologies, que asimila el efecto directo con "consecuencia inmediata". En su opinión, dicha interpretación se centra en un factor único de separación temporal y espacial entre conducta y efecto, en circunstancias que una interpretación fidedigna de la ley admite un nexo causal más amplio y razonablemente próximo. Sin embargo, aplicando este criterio, desestimó la acción presentada porque el perjuicio alegado por el demandante precedía a los efectos en suelo estadounidense, siendo que "un efecto nunca precede a una causa". Además, esta última jurisprudencia

\footnotetext{
${ }^{40}$ Corte de Apelaciones de Estados Unidos para el Séptimo Circuito, 26.11.2014, Rol No 14-8003. Cfr. Fiebig, 2015, pp. 2A a $11 \mathrm{~A}$.

${ }^{41}$ En Estados Unidos, la persecusión de conductas anticompetitivas a nivel federal está a cargo del Departamento de Justicia (DOJ) y de la Comisión Federal de Comercio (FTC). El primero puede ejercer acciones civiles y penales, mientras que a la segunda corresponde ejercer acciones civiles y pronunciarse por operaciones de concentración.

${ }^{42}$ Corte de Apelaciones de Estados Unidos para el Séptimo Circuito, 27.6.2012, Rol No 08 C 6910, MDI. N 1996.

${ }^{43}$ Corte de Apelaciones de Estados Unidos para el Segundo Circuito, 4.6.2014, Rol N 13-2280.
} 
ha sido objeto de críticas por las incertidumbres y riesgos de afectación que causa en jurisdicciones extranjeras ${ }^{44}$.

Por su parte, la Corte de Justicia Europea presenta una evolución similar a la de Estados Unidos, pues pasa de exigir afectación directa del mercado concernido a permitir sanciones por afectación de mercados conexos al de la conducta anticompetitiva.

Un primer antecedente del primer período es el caso Béguelin ${ }^{45}$, sobre acuerdo de distribución exclusiva impuesto por un proveedor extranjero a una empresa europea, en que la Corte sancionó la conducta sin siquiera mencionar los hechos del caso, "dado que el acuerdo produce sus efectos en el territorio del mercado común”.

Otro ejemplo es la sentencia en el caso Absltröm ${ }^{46}$, sobre colusión de productores de pasta de madera domiciliados fuera de la Comunidad que restringió la competencia dentro de ella. La Corte justificó el juzgamiento de ilícitos acordados en el extranjero para ser ejecutados en suelo europeo señalando que si se exigiera la territorialidad del acuerdo "ello nos conduciría claramente a facilitar a las empresas un medio fácil para sustraerse a dichas prohibiciones. Lo que es determinante, por tanto, es el lugar en el que el acuerdo colusorio se ejecuta".

Es significativo mencionar también el caso Gencor ${ }^{47}$, sobre concentración de dos empresas productoras mundiales de platino en Sudáfrica que vendían parte de su producción en el mercado europeo. La CEJ estableció que la Comisión solo podría controlar operaciones de concentración extranjeras que produzcan un efecto "inmediato, sustancial y previsible” en el mercado europeo. Aclaró que el efecto inmediato no consiste necesariamente en un atentado a la libre competencia, sino que basta con generar las condiciones para que este sea posible y económicamente racional. Por su parte, el carácter sustancial del efecto es definido en términos de duración y volumen relevante de ventas. Además, señaló que la operación debe estar directamente orientada a la Comunidad.

Sin perjuicio de lo anterior, la jurisprudencia más reciente de la CEJ ha evolucionado desde la doctrina de la "implementación" del acuerdo hacia la de los "efectos calificados", en virtud de ello, es posible justificar, además, sanciones a conductas anticompetitivas extraterritoriales en la venta de insumos también en el extranjero, pero destinados a la elaboración de productos que serán vendidos en Europa. De esta forma, establece un precedente relevante de sanción por afectar mercados europeos conexos a aquel extranjero en que incide la conducta colusiva.

Un primer antecedente es el caso Innolux ${ }^{48}$, en que dos empresas asiáticas integradas verticalmente se coludieron para fijar el precio de las pantallas de cristal líquido

\footnotetext{
${ }^{44}$ Cfr. Harvard Law Review, 2014, pp. 759 a 766.

45 CEJ, 25.11.1971, Rol Caso 22/71.

46 Absltröm Osakeybtiö, CEJ, 27.9.1988, casos acumulados Rol No 89/85. Cfr. BaLlarino, T., "L'art. 6
} del Regolamento Roma II e Il Diritto antitrust comunitario: conflicto di leggi e principio territorialistico”, RDI, 2008, pp. 65-78.

${ }^{47}$ CEJ, 25.3.1999, Rol asunto T-102/96.

${ }^{48}$ CEJ, 9.7.2015, Rol Caso C-231/14 P. 
con matriz activa (LCD), insumo que era vendido a manufacturadores de monitores en Europa y en otras partes del mundo que también vendían en Europa. En el caso de estos últimos, la conducta colusiva incidía primero en un mercado extranjero de insumos, para luego producir efectos en Europa solo mediante la venta de monitores. Por esta razón, Innolux alegó que la conducta no era sancionable porque incidió directamente en un mercado fuera del territorio europeo. Sin embargo, la Corte confirmó la competencia para sancionar atendido que la colusión determinó un alza del precio del producto final que afectó directamente el mercado del viejo continente. A su juicio, el acuerdo fue "implementado" igualmente en Europa porque tenía el objeto y efecto de restringir la competencia en su mercado interno.

Una doctrina similar adoptó la Corte en el caso $L G$ Electronics ${ }^{49}$, sobre colusión en el mercado de los tubos de rayos catódicos, el acuerdo celebrado en el extranjero versaba por insumos vendidos fuera de Europa para la fabricación de monitores y televisores destinados a dicho continente. La empresa sancionada alegó que esta afectación indirecta del mercado europeo impedía el ejercicio de poderes sancionatorios, pues no se cumplía el requisito de "inmediatez" de la lesión. Sin embargo, la Corte validó la sanción porque consideró que la colusión sobre el insumo afectó igualmente la libre competencia respecto del producto final en Europa. Correspondía, por tanto, sancionarla pese a que el mercado afectado en Europa estaba separado de aquel en que se produjo la infracción.

Otro ejemplo es el caso Intel $^{50}$, uno de sus últimos pronunciamientos respecto de la materia. La empresa fue sancionada por haber pagado rebates de exclusividad a dos fabricantes de computadores establecidos en Asia para que atrasaran o cancelaran la oferta de productos que utilizaran microprocesadores de sus competidores. Su primera alegación consistió en la incompetencia de la Comisión porque la conducta había sido ejecutada en otro continente. Sin embargo, la Corte sostuvo que la Comisión era competente para sancionar atendido que la conducta foránea formaba parte de una "estrategia de conjunto destinada a cerrar el acceso de AMD a los canales de venta más importantes del mercado", y que bastaba la probabilidad de aquello para cumplir con el requisito de previsibilidad de afectación a la libre competencia en Europa.

De esta forma, como se aprecia, la jurisprudencia de Estados Unidos y de la Unión Europea ha evolucionado hacia una interpretación de "efecto directo" que admite un nexo causal mediato entre la conducta anticompetitiva y el territorio afectado, en aquellos casos en que esta incide inmediatamente en un mercado conexo extranjero.

\footnotetext{
${ }^{49}$ CEJ, 9.9.2015, Rol T-91/13.

${ }^{50}$ CEJ, 6.9.2017, Rol C-413/14 P.
} 


\section{El JUZGAMiento Limitado POR DEFERENCIA A LA JURISDICCIÓN EXTRANJERA O COMITY}

La sanción de conductas extranjeras que atentan en contra de la competencia en suelo nacional se ha visto moderada en algunos casos por una doctrina intermedia que procura conciliar los intereses estatales involucrados con los principios consuetudinarios del derecho internacional público. Esta exige como título justificativo de la jurisdicción nacional la producción de efectos de magnitud suficiente en relación con los causados en otras jurisdicciones ${ }^{51}$.

En Estados Unidos, esta posición está representada por el citado fallo de la Corte de Apelaciones del Noveno Circuito en Timberlane. Precisó que la doctrina de los efectos sustantivos sería insuficiente para confirmar la jurisdicción norteamericana, porque no considera en plenitud los intereses de otras naciones y la real vinculación de los actores del mercado con Estados Unidos, por ejemplo, en materia de nacionalidad. Es por esta razón que la sentencia incorporó una pregunta adicional al test de los efectos: ¿Son los vínculos e intereses de Estados Unidos en el caso, incluyendo el grado de afectación de su comercio exterior, lo suficientemente significativos -en relación con los de otros países- como para justificar el ejercicio de jurisdicción extraterritorial?

Esta pregunta se refiere, principalmente, al deber de cortesía, buena fe, deferencia o comity de los tribunales de un Estado hacia la jurisdicción territorial y las sentencias de los tribunales de otro Estado.

Los fundamentos de este deber en asuntos de libre competencia fueron mencionados en la sentencia del caso Empagran ${ }^{52}$. En primer lugar, se basa en la necesidad de respetar los intereses soberanos legítimos de otras naciones, con el fin de armonizar leyes potencialmente conflictivas en un mundo comercial altamente interdependiente. En segundo lugar, el lenguaje y la historia de la reforma que incorporó el comercio exterior en la Ley Sherman sugieren que solo tuvo por finalidad clarificar, tal vez limitar, pero no expandir de modo significativo el ámbito de aplicación de dicha ley.

\section{Conflicto de leyes y de jurisdicción}

En cuanto a la aplicación misma del criterio de comity, la Corte sostuvo en Timberlane que la ponderación de los factores obedece a una "regla de razón jurisdiccional" propia del conflicto de leyes en el derecho internacional. Esta valoración considera elementos como

"el grado de conflicto con el derecho o política extranjeros, la nacionalidad o vínculo jurídico de las partes y el domicilio o principal asentamiento de los negocios y empresas, la posibilidad real de que la exigencia judicial sea obedecida, la importancia relativa de los efectos en Estados Unidos en comparación a otros países, hasta qué

\footnotetext{
${ }^{51}$ Cfr. Burnett, 2004, p. 555; Casey, 2005, p. 585.

52 Corte Suprema de Estados Unidos, 14.6.2004, Rol No 03-724.
} 
punto existe el propósito explícito de dañar o afectar el comercio de Estados Unidos, la previsibilidad de tal efecto, y la importancia relativa de la infracción en Estados Unidos en comparación con la misma conducta en el exterior".

Otro elemento a considerar en el análisis de comity es el posible conflicto de leyes entre dos países con competencias concurrentes. La Corte Suprema de Estados Unidos tuvo oportunidad de precisar este concepto en el caso Hartford, citado anteriormente. Las compañías reaseguradoras inglesas reconocieron la competencia del tribunal americano, pero defendieron la preeminencia de la jurisdicción británica en virtud del principio de comity internacional. En particular, mencionaron un grave conflicto entre la Ley Sherman y el derecho británico de libre competencia, porque este no sancionaba la conducta denunciada.

Sin embargo, la Corte Suprema de Estados Unidos declaró el conflicto inexistente, ya que dicha conducta no era exigida por ninguna de las leyes. A su juicio, el verdadero conflicto -que podría determinar una declinatoria de competencia- se produce cuando la conducta es castigada por un Estado y exigida - no simplemente permitida- por otro, de modo que el respeto por ambos derechos se hace imposible. En cambio, si la conducta no es obligatoria en ninguno de los ordenamientos, el cumplimiento de ambos es siempre posible, razón por la que no existiría conflicto alguno.

Un criterio similar adoptó la CEJ en el citado caso Absltröm ante el argumento de los productores estadounidenses de que la colusión les era permitida por su Ley WebbPomenere. La sentencia sostuvo que dicha ley "se limita a exceptuar de la aplicación de las leyes en materia de prácticas colusorias americanas la celebración de acuerdos colusorios sobre exportación, sin imponer la obligación de celebrar tales acuerdos”.

Ahora bien, en relación con la prueba del derecho extranjero, la jurisprudencia se ha mostrado deferente, obviamente, a lo que puedan declarar en el juicio los estados a los que pertenecen las empresas acusadas ${ }^{53}$.

Una variante de esta última jurisprudencia dice relación con lo que se entiende por "conflicto de jurisdicción” con otros estados. Así, por ejemplo, el citado caso Hartford sostiene una interpretación minimalista, en virtud de ello, solo hay conflicto cuando la conducta considerada anticompetitiva en un Estado es forzada por otro Estado. La sentencia del caso Empagran, en cambio, define el conflicto en términos de mera coexistencia de poderes sancionadores respecto de conductas extranjeras.

La interpretación maximalista orientó, por ejemplo, el fallo de la Corte en el citado caso Daishowa. La importadora japonesa de chips de madera, demandada en Estados Unidos, opuso la excepción de incompetencia del tribunal por existir un conflicto de leyes extranjeras, y porque el deber de comity exigía garantizar a los importadores japoneses la misma inmunidad de jurisdicción de que gozan los exportadores norteamericanos

${ }^{53}$ In re Vitamin C Antitrust Litigation, Corte de Apelaciones de Estados Unidos para el Segundo Distrito, 20.9.2016, Rol No 13-4791-cv. Una doctrina similar se aplico en el citado caso Absltröm. 
bajo la Ley Webb-Pomenere ${ }^{54}$. Sin embargo, la Corte desestimó un posible conflicto de normas y aclaró que la citada ley no eximía a importadores nacionales de fijaciones de precios "injustas u opresivas" o de conductas "inmorales para la filosofía americana de libre competencia" 55 , razón por la que la reciprocidad no era aplicable. Además, fundó su competencia en la falta de interés de Japón para sancionar el ilícito, la falta de medidas de protección en el derecho japonés, la gravedad de las conductas imputadas y el daño que podrían causar.

Sin perjuicio de lo anterior, conviene señalar que en algunos casos los tribunales extranjeros han invocado el deber de comity para dejar sin efecto la jurisdicción norteamericana. En Imperial Chemical ${ }^{56}$, una empresa británica fue condenada en Estados Unidos a enajenar algunas patentes. Sin embargo, la compañía había celebrado un contrato de licencia exclusiva de sus patentes en Inglaterra con una empresa ajena al juicio. Por esta razón, una Corte de Apelaciones inglesa prohibió la enajenación y ordenó el cumplimiento del contrato en resguardo del derecho inglés ${ }^{57}$.

Así también, en Laker Airways ${ }^{58}$, una aerolínea británica demandó a competidoras nacionales y europeas ante un tribunal de Estados Unidos por precios predatorios en vuelos internacionales que la condujeron a la quiebra. Las acusadas alegaron que el litigio debía ser conocido por tribunales ingleses en atención a la nacionalidad del demandante o principio de la "nacionalidad primordial" (paramount nationality). Además, invocaron la inaplicabilidad del derecho de libre competencia norteamericano a empresas británicas en razón del tratado bilateral de Bermuda $\mathrm{II}^{59}$. Por esta razón, solicitaron a la justicia del Reino Unido ordenar a Laker desistirse de su demanda. Esta solicitud fue acogida definitivamente una vez que el Secretario de Estado para el Comercio y la Industria del Reino Unido, en ejercicio de los poderes conferidos por la ley de protección de intereses comerciales ${ }^{60}$, prohibió a las personas con negocios en el Reino Unido cumplir las resoluciones de libre competencia de tribunales estadounidenses relativas a aerolíneas británicas $^{61}$.

${ }^{54}$ Ley Webb-Pomenere, 1918, que exime de las sanciones de la Ley Sherman a la asociación formada con el solo propósito de realizar exportaciones, en la medida que dicha actividad no afecte la competencia o comercio interior de Estados Unidos.

${ }^{55}$ La sentencia citó al respecto los casos United States v. United States Alkali Export Ass'n, Corte de Distrito de Nueva York S.D., 12.8.1949, Rol Civ. No 24-464; y United States v. Minnesota Mining and Manufacturing, Corte de Distrito de Massachusetts, 13.9.1950, Rol Civ. A. No 8119.

56 Corte de Distrito de Nueva York S.D., 16.5.1952, Rol desconocido.

${ }^{57}$ British Nylon Spinners Ld v. Imperial Chemical Industries LD, Corte de Apelaciones de Inglaterra, 15.10.1952, Rol No 3747.

58 Corte de Apelaciones de Estados Unidos para el Distrito del Circuito de Columbia, 6.3.1984, Rol $\mathrm{N}^{\circ}$ s 83-1280, 83-1281.

${ }^{59}$ Consolidated Air services Agreement between the government of the United States of America and the Government of the United Kingdom of Great Britain and Northern Ireland, 1977.

${ }^{60}$ Protection of Trading Interests Act 1980, 1980 (Chapter 11).

${ }^{61}$ Secretario de Estado para el Comercio y la Industria, 23.6.1983. Cfr. CANnON, 1985, pp. 63-87. 
Sin embargo, la Corte de Apelaciones del distrito de Columbia confirmó la competencia del tribunal de acuerdo con la doctrina de los efectos. Señaló que, aunque fueran invisibles, las consecuencias radiantes de los actos extranjeros causan una lesión económica no menos tangible que el daño de "balas asesinas" disparadas desde el otro lado de la frontera o las llamadas telefónicas de ladrones que roban recursos financieros desde el exterior.

En lo que respecta al test de comity, la Corte fundó la preeminencia de la jurisdicción americana por sobre la británica en cinco razones. Primero, el alza de precios en los pasajes afectaría especialmente a los consumidores de Estados Unidos. Además, los acreedores afectados por la quiebra del demandante eran principalmente norteamericanos. Ellos tienen derecho a confiar en la habilidad del país para proteger sus créditos. En tercer lugar, las aerolíneas demandadas desarrollaban importantes operaciones en Estados Unidos sujetas a la legislación y tribunales del país anfitrión. Cuarto, el foro inglés no ofrecía la solución de indemnización de perjuicios triplicada (treble damages) solicitada por el demandante. Finalmente, la regla de la "nacionalidad primordial" no había sido reconocida por el derecho internacional, desconocía absolutamente los intereses territoriales y era imposible de administrar en la práctica. A modo de ejemplo, existen al menos cinco tipos de criterios para determinar la nacionalidad de una persona jurídica, así como la nacionalidad de sus accionistas, el estado en que fue incorporada, u otros vínculos corporativos con un foro en particular.

Por último, parte de la doctrina ha sugerido abordar los problemas de cortesía mediante acuerdos bilaterales ${ }^{62}$ o multilaterales ${ }^{63}$, aunque con escasos resultados y críticas variadas ${ }^{64}$. En el caso concreto de Estados Unidos y la Unión Europea, el primer Acuerdo de Cooperación es de 1991. Sin embargo, este fue impugnado por Francia y dejado sin efecto por la CEJ, que estimó que la Comisión Europea carecía de competencia para celebrarlo ${ }^{65}$. Posteriormente, fue rectificado por una decisión conjunta del Consejo Europeo y la Comisión en $1995^{66}$.

Este acuerdo establece deberes de comity positivo (art. V) y negativo (art. VI). El primero consiste en pedirle a la parte extranjera donde se estarían realizando conductas anticompetitivas que afectan intereses de la contraparte que inicie las actividades de sanción correspondiente. El segundo exige a ambas partes que el ejercicio de sus poderes de policía considere los intereses de su contraparte con el fin de evitar conflictos. Para tal efecto, deberán considerar factores como la importancia de las conductas en el territorio, el orden jurídico y la afectación de los consumidores. Posteriormente, el deber de comity

${ }^{62}$ Cfr. CHANG, 1993, p. 295.

${ }^{63}$ Basedow, 1999-2000, p. 1037; BATZ, 2007, p. 65.

${ }^{64}$ Cfr. Shank, 1996, p. 155; y Mehra, 1999, p. 191.

${ }^{65}$ France v. Commission, CEJ, 9.8.1994, Rol Caso C-327/91.

${ }^{66}$ Acuerdo entre las Comunidades Europeas y el Gobierno de Estados Unidos de América relativo a la aplicación de sus normas de competencia, 1995. 
positivo fue precisado en un acuerdo de 1998 que permite a cada parte aplicar sanciones que sean acordes con el derecho de libre competencia de su contraparte (art. III) ${ }^{67}$.

\section{Participación de Estados extranjeros en el ilícito}

Adicionalmente, la Corte Suprema de Estados Unidos ha fijado precedentes acerca de acciones empresariales realizadas con la colaboración de un Estado foráneo para producir efectos anticompetitivos en su territorio. En la totalidad de los casos, las sentencias han confirmado la competencia para juzgar la responsabilidad de la empresa en los hechos, pero han declinado conocer siquiera de la participación del Estado foráneo en los mismos.

En Sisal ${ }^{68}$, por ejemplo, el mercado del sisal en México era imperfecto porque la legislación establecía un comprador estatal único de los proveedores y un agente exclusivo de exportación: la empresa demandada. La Corte fue clara en señalar que su competencia se extendía únicamente a la responsabilidad de la empresa en los hechos y no a la del Estado, aunque este hubiese colaborado o facilitado la conducta por medio de sus normas o actos. Un criterio similar fue aplicado en Continental ${ }^{69}$, en que una empresa estadounidense fue acusada de conspirar para restringir y monopolizar el mercado del vanadio producido en Estados Unidos, inter alia, mediante actos de su sociedad filial en Canadá que actuaba como agente exclusivo del gobierno de dicho país. La Corte sostuvo que los tribunales estadounidenses eran competentes para juzgar únicamente la conducta de la sociedad.

Sin perjuicio de lo anterior, la Corte ha reconocido la posibilidad de no sancionar a empresas involucradas en prácticas anticompetitivas en el extranjero cuando su conducta haya sido impuesta por el Estado soberano foráneo. Así, por ejemplo, la negativa a vender petróleo crudo venezolano a una empresa no fue considerada un acto ilegal de restricción de comercio porque se fundaba en un boicot impuesto por el gobierno venezolano ${ }^{70}$.

El citado fallo en Timberlane es también relevante porque desarrolla el marco conceptual de la incompetencia de los tribunales norteamericanos para juzgar actos estatales extranjeros que afecten la libre competencia. Se funda para ello en la doctrina del "acto de Estado" aplicada en Banco Nacional de Cuba ${ }^{71}$. En este caso, la Corte Suprema concluyó que la doctrina no se basa en la soberanía del país extranjero, ni en el derecho internacional o la Constitución de Estados Unidos, así como lo sugería una jurisprudencia

${ }^{67}$ Acuerdo entre las Comunidades Europeas y el gobierno de Estados Unidos de América acerca de la observancia de los principios de cortesía positiva en la aplicación de sus normas de competencia, 1998. Esta sería, además, la solución de los tratados internacionales suscritos por Chile con Estados Unidos y la Unión Europea, particularmente en relación con el alcance de las potestades persecutorias de la Fiscalía Nacional Económica. Disponibles en https:/www.fne.gob.cl/internacional/acuerdos-de-cooperacion/

68 Corte Suprema de Estados Unidos, 16.5.1927, Rol N² 200.

${ }^{69}$ Corte Suprema de Estados Unidos, 25.6.1962, Rol No 304.

${ }^{70}$ Corte de Distrito de Delaware, 7.1.1970, Rol N ${ }^{\circ}$ Civ. A. N ${ }^{\circ} 2808$.

${ }^{71}$ Corte Suprema de Estados Unidos, 23.3.1964, Rol No 16. 
anterior $^{72}$. Más bien, deriva del principio de no intervención judicial en la conducción de las relaciones exteriores por parte de los poderes políticos.

Sin embargo, la Corte en Timberlane reconoció que la doctrina del "acto de Estado" no rige de modo absoluto o inflexible. La intensidad de su aplicación dependerá de la importancia del asunto para las relaciones internacionales de Estados Unidos. Así, por ejemplo, no sería aplicable frente a meras expresiones de funcionarios estatales extranjeros no contenidas en actos jurídicos legislativos o administrativos. Tampoco se extendería, por regla general, a las sentencias judiciales dictadas en litigios entre privados sin injerencia formal del gobierno. En el caso concreto, la Corte precisó que el hostigamiento judicial no fue promovido por el gobierno de Honduras sino por sujetos particulares, razón por la que las sentencias no obedecían a actos de soberanía sujetos a la doctrina del "acto de Estado".

Otro acto público no sujeto a la doctrina del "acto de Estado" es el otorgamiento de una patente comercial. En Mannington ${ }^{73}$, una empresa norteamericana fue acusada de obtener patentes extranjeras de modo fraudulento, afectando de este modo a sus competidoras de igual nacionalidad. La demandada alegó que el otorgamiento de patentes por órganos extranjeros es un acto de Estado exento de control. Sin embargo, la Corte del Tercer Circuito declaró que "el otorgamiento de patentes para instalar pisos flotantes no es el tipo de decisión soberana que podría ser de especial preocupación para el poder ejecutivo en la conducción de sus asuntos internacionales”. Por esta razón, el tribunal ejerció igualmente competencia respecto de las conductas anticompetitivas de la empresa.

\section{JUZGAMIENTO LIMITADO POR EL PRINCIPIO NON BIS IN IDEM EN RELACIÓN CON LA JURISDICCIÓN EXTRANJERA}

Finalmente, surge la interrogante de si un agente económico que ha sido juzgado en otros países puede ser condenado por los mismos hechos en Estados Unidos o Europa; en otras palabras, si es posible o no aplicar la doctrina del non bis in idem para limitar la imposición de sanciones en estos casos, en el supuesto de identidad de infracción y de sujeto infractor. La pregunta no se refiere, por tanto, al doble juzgamiento a nivel federal y estadual en Estados Unidos, ni al doble juzgamiento a nivel comunitario y de miembros de la Comunidad en Europa.

Una primera aproximación doctrinal es el principio de "soberanía dual" (dual sovereignty), de origen estadounidense y propio de jurisdicciones federales, en virtud de ello, el criterio de non bis in idem no se aplica a ilícitos que afectan soberanías independientes, pues no concurre el presupuesto de identidad de soberanía necesario para aplicarlo.

\footnotetext{
${ }^{72}$ Underbill v. Hernández, Corte Suprema de Estados Unidos, 29.11.1897, Rol No 36.

${ }^{73}$ Corte de Apelaciones de Estados Unidos para el Tercer Circuito, 3.4.1979, Rol No 78-1845.
} 
Además, siendo el ilícito un atentado a la soberanía de cada Estado, no corresponde que uno asuma la protección de la soberanía del otro ${ }^{74}$.

La jurisprudencia de la CEJ confirma la improcedencia del non bis in idem en casos de libre competencia, aunque sustentada en la falta de identidad del bien jurídico protegido. Específicamente, la diferencia de territorios y mercados no permite configurar la identidad de intereses necesaria para aplicar la garantía de la doble incriminación ${ }^{75}$. Sin perjuicio de lo anterior, la CEJ ha señalado que, en virtud del principio de proporcionalidad, "si bien no cabe excluir que la Comisión tenga en cuenta multas anteriormente impuestas por las autoridades de Estados terceros, no está, sin embargo, obligada a hacerlo" ${ }^{\text {76. }}$.

De esta forma, la jurisprudencia de Estados Unidos y de Europa confirma que, hasta ahora, el principio non bis in idem no puede ser invocado para evitar la aplicación de sanciones por el mismo hecho en varias jurisdicciones, aunque sí tal vez para reducir su monto.

\section{Conclusiones}

De acuerdo con lo expuesto, es posible deducir las siguientes conclusiones del texto:

1. En materia de juzgamiento y sanción de conductas anticompetitivas extraterritoriales en Estados Unidos y la Unión Europea, es posible advertir el ejercicio de una competencia basada en la territorialidad de su implementación o efectos, aunque sometido en algunos casos a elementos moderadores de comity o deferencia hacia jurisdicciones extranjeras.

2. El carácter "doméstico" o "foráneo" de una conducta en la que participan sujetos nacionales y extranjeros dependerá de la prevalencia del conjunto de factores, entre los que destacan la nacionalidad de los participantes, sus actividades, mercados objetivos y efectos, así como la existencia de empresas filiales.

3. Los efectos territoriales que debe producir la conducta para lesionar la competencia doméstica deben ser directos, sustanciales y razonablemente previsibles. El carácter "directo" del efecto admite también la afectación directa de dicho mercado debido a su relación con un mercado conexo extraterritorial en el que incidió la conducta.

4. La jurisprudencia de Estados Unidos ha declarado que, en caso de conductas anticompetitivas realizadas con la colaboración de un estado foráneo, solo procede juzgar la responsabilidad de la empresa.

${ }^{74}$ Cfr. Heath v. Alabama, Corte Suprema de Estados Unidos, 3.12.1985, Rol No 84-5555; y United States v. Lanza, Corte Suprema de Estados Unidos, 11.12.1922, Rol No 39.

75 Tokai Carbon con Comisión, CEJ, Rol 29.6.2006, C-301/04 p.

76 Showa Denko KK con Comisión, CEJ, 29.6.2006, Rol Caso C-289/04 P; Schunk GmbH con Comisión, CEJ, 8.10.2008, Rol T-69/04. Para un análisis sistemático de la jurisprudencia europea, véase CALvo y CAnedo, 2006, pp. 11 a 39. 
5. Finalmente, la jurisprudencia de Estados Unidos y de Europa confirma que, hasta ahora, el principio non bis in idem no puede ser invocado para evitar la aplicación de sanciones por el mismo hecho en terceros países, aunque sí tal vez para reducir su monto.

\section{BiBLIOGRAFÍA}

BASEDow, Jurgen, 1999-2000: "International Antitrust: From Extraterritorial Application to Harmonization", Louisiana Law Review, Vol. 60.

Batz, Marie T., 2007, "Comparative Analysis of United States and European Union Jurisdiction in Extraterritorial Antitrust Law \& the Need for International Standards", Duquesne Business Law Journal, Vol. 9.

Beckler, R., y Kirtland, M., 2003: "Extraterritorial Application of U.S. Antitrust Law: What Is a 'Direct, Substantial, and Reasonably Foreseeable Effect' Under the Foreign Trade Antitrust Improvements Act?", Texas International Law Journal, Vol. 38.

Blythe, M.A., 1983: "The extraterritorial impact of the anti-trust laws: protecting British trading interests", The American Journal of Comparative Law, Vol. 31.

BurnetT, Susan, 2004: "E.U.S. Judicial Imperialism Post Empagran v. F. Hoffmann-Laroche - Conflicts of Jurisdiction and International Comity in Extraterritorial Antitrust", Emory International Law Review, Vol. 18, Issue 2.

Calvo C., Alfonso y Canedo, María P., 2006: "Non bis in idem en Derecho antitrust", Estudios de Deusto, Segunda época, Vol. 54.

Cannon, Robert, 1985: "Laker Airways and the courts: a new method of blocking the extraterritorial application of U.S. antitrust laws", Journal of Comparative Business and Capital Market Law, 7.

Casey, Stephanie A., "Balancing Deterrence, Comity Considerations, and Judicial Efficiency: The Use of the D.C. Circuit's Proximate Cause Standard for Determining Subject Matter Jurisdiction over Extraterritorial Antitrust Cases", American University Law Review, Vol. 55, Issue 2.

Chang, S.W., 1993: "Extraterritorial Application of U.S. Antitrust Laws to Other Pacific Countries: Proposed Bilateral Agreements for Resolving International Conflict within the Pacific Community", Hastings International Comparative Law Review, Vol. 16.

DAM, Kenneth, 1994: "Extraterritoriality in an age of Globalization: The Hartford Fire Case", Supreme Court Review, Vol. 1993.

FERnANDES, Siddharth, 2005: "F. Hoffman-Laroche, Ltd. v. Empagran and the Extraterritorial Limits of United States Antitrust Jurisdiction: Where Comity and Deterrence Collide", Connecticut Journal of International Law, Vol. 20, Issue 2.

Fiebig, Andre, 2015: "Import Commerce and the Foreign Trade Antitrust Improvements Act", Northwestern Journal of International Law E Business, Vol. 35, № $21 \mathrm{~A}$.

Gascón InCHausti, Fernando, 2017: Reconocimiento y ejecución de resoluciones extranjeras en el nuevo Reglamento de Bruselas I Bis, Valencia, Tirant lo Blanch.

Gilsanz Usunaga, Javier, 2010: El proceso civil estadounidense. La tutela judicial cautelar, Pamplona: Aranzadi.

Gupta, V., 1996: “After Hartford Fire: Antitrust and Comity”, Georgetown Law Journal, Vol. 84, 1996. 
Harvard Law Review, 2014: "Lotes Co. v. Hon Hai Precision Industry Co.”, Harvard Law Review, $\mathrm{N}^{\circ} 128$.

Menra, Salil, 1999: "Extraterritorial Antitrust Enforcement and the Myth of International Consensus", Duke Journal of Comparative E International Law, Vol. 10.

Ministerio de Relaciones Exteriores, 2018: Reporte Annual Comercio Exterior de Chile, Dirección General de Relaciones Económicas Internacionales, Departamento de Estudios.

Odudu, O., Bailey, D., 2014, "The single economic entity doctrine in EU Competition law", Common Market Law Review, Vol. 51 (6).

ONGMAN, John, 1977: "Be no longer chaos: constructing a normative theory of the Sherman Act's extraterritorial jurisdictional scope”, Northwestern University Law Review, Vol. 71, № 6.

OrmaZABAL SÁnchez, Guillermo, 2016: La brecha procesal civil entre EE.UU. y Europa, Valencia, Tirant lo Blanch.

Prete, Luca, 2018: "On implementation and Effects: The Recent cCase-law on the Territorial (or Extraterritorial?) Application of EU Competition Rules”, Journal of European Competition Law E Practice.

SAmIE, Najeeb, 1981, "Extraterritorial Enforcement of U.S. Antitrust Laws: the British Reaction”, Maryland Journal of International Law, Vol. 7, Issue 1.

SHank, Robert, 1996: “The Justice Department's Recent Antitrust Enforcement Policy: Toward a 'Positive Comity' Solution to International Competition Problems?", Vanderbilt Journal of Transnational Law, Vol. 29.

Torremans, P., 1996: "Extraterritorial Application of E.C. and U.S. Competition Law”, European Law Review, Vol. 21.

Signes de Mesa, J.I., Fernández, I., y Fuentes, 2013: M., Derecho de la Competencia, Madrid, Thomson Reuters.

Trenor, J.A., 1995: "Jurisdiction and the Extraterritorial Application of Antitrust Laws after Hartford Fire", University of Chicago Law Review, Vol. 62.

United States Congress, 1982: House of Representatives Report No 97-686, 9-10, como fue impresa en 1982 U.S.C.C.A.N. 2487, 2487-2488, 2494-2495.

\section{Normas jurídicas citadas}

Acuerdo entre las Comunidades Europeas y el Gobierno de Estados Unidos de América relativo a la aplicación de sus normas de competencia, publicado en 1995, Diario Oficial de la Comunidad Europea, L 94/95, corregido por L 131/38.

Acuerdo entre las Comunidades Europeas y el Gobierno de Estados Unidos de América sobre la observancia de los principios de cortesía positiva en la aplicación de sus normas de competencia, publicado en 1998, Diario Oficial de la Comunidad Europea, L 173/26.

CONSOLIDATED Air services Agreeement between the government of the United States of America and the Government of the United Kingdom of Great Britain and Northern Ireland, 23 de julio de 1977, 28 U.S.T. 5376, T.I.A.S. $\mathrm{N}^{\circ} 8641$.

THE Foreign Trade Antitrust Improvements Act of 1982, A bill to amend the Sherman Act, the Clayton Act, and the Federal Trade Commission Act to exclude from the application of such acts certain conduct involving exports, publicada el 8 de marzo de 1982, 96 Stat. 1246,15 U.S. C. $\$ 6^{a}$.

THE Sherman Antitrust Law, To protect trade and commerce against unlawful restraints and monopolies, publicada el 2 de julio de 1890, 26 Stat. 209 (1887-1891) Chapter 647, 51 Congress, Session 1. 
THE Webb-Pomenere Act, An act to promote export trade, and for other purposes, publicada el 10 de abril de 1918, 15 U.S.C. $\S \S 61-68$.

THE Protection of Trading Interests Act 1980, An Act to provide protection from requirements, prohibitions and judgments imposed or given under the laws of countries outside the United Kingdom and affecting the trading or other interests of persons in the United Kingdom, publicada el 20 de marzo de 1980.

U.S. Antitrust Measures, Order of The Secretary of State for Trade and Industry Protection of Trading Interests, publicada el 23 de junio de 1983.

\section{Jurisprudencia citada}

Cámara de los Lores del Reino Unido. Sentencia de 1 de diciembre de 1977, Rol No 235 $\left(\mathrm{N}^{\circ} 1\right.$ y $\left.\mathrm{N}^{\circ} 2\right)$.

Corte de Apelaciones de Estados Unidos para el Cuarto Distrito. Sentencia de 30 de julio de 2002, Rol No 01-1894.

Corte de Apelaciones de Estados Unidos para el Décimo Circuito. Sentencia de 14 de octubre de 1981, Rol No 79-1999.

Corte de Apelaciones de Estados Unidos para el Distrito del Circuito de Columbia, Sentencia de 6 de marzo de 1984, Rol No s 83-1280, 83-1281.

Corte de Apelaciones de Estados Unidos para el Noveno Circuito. Sentencia de 11 de agosto de 2004, Rol No 02-16472.

Corte de Apelaciones de Estados Unidos para el Noveno Circuito. Sentencia de 27 de diciembre de 1976, Rol Nos. 74-2142, 74-2354, 74-2812 y 74-2813549.

Corte de Apelaciones de Estados Unidos para el Segundo Circuito. Sentencia de 12 de marzo de 1945, Rol No 144.

Corte de Apelaciones de Estados Unidos para el Segundo Circuito. Sentencia de 4 de junio de 2014, Rol N 13-2280.

Corte de Apelaciones de Estados Unidos para el Segundo Distrito. Sentencia de 20 de septiembre de 2016, Rol No 13-4791-cv.

Corte de Apelaciones de Estados Unidos para el Séptimo Circuito. Sentencia de 15 de febrero de 1980, Rol no citado.

Corte de Apelaciones de Estados Unidos para el Séptimo Circuito. Sentencia de 26 de noviembre de 2014, Rol No $14-8003$.

Corte de Apelaciones de Estados Unidos para el Séptimo Circuito. Sentencia de 27 de junio de 2012, No 08 C 6910, Rol MDI. No 1996.

Corte de Apelaciones de Estados Unidos para el Tercer Circuito. Sentencia de 3 de abril de 1979, Rol No 78-1845.

Corte de Apelaciones de Estados Unidos para el Tercer Circuito. Sentencia de 8 de septiembre de 2000, Rol No 99-5931.

Corte de Apelaciones de Inglaterra. Sentencia de 15 de octubre de 1952, Rol No 3747.

Corte de Distrito de California N.D. Sentencia de 20 de octubre de 1978, Rol C-70-852 SAW.

Corte de Distrito de Delaware. Sentencia de 7 de enero de 1970, Rol No Civ. A. N 2808.

Corte de Distrito de Delaware. Sentencia de 26 de septiembre de 2006, Rol MDL 0517174JF, CIV.A.05-441-JJF.

Corte de Distrito de Massachusetts. Sentencia de 13 de septiembre de 1950, Rol Civ. A. $\mathrm{N}^{\circ} 8119$.

Corte de Distrito de Nueva York S.D. Sentencia de 12 de agosto de 1949, Rol Civ. No 24-464. Corte de Distrito de Nueva York S.D. Sentencia de 16 de mayo de 1952, Rol desconocido. 
Corte de Distrito de Nueva York S.D. Sentencia de 21 de enero de 1983, Rol No 82 Civ. 4269 (KTD).Corte de Distrito de Nueva York S.D., Sentencia de 29 de enero 2001, Rol No 00Civ.6322 (LAK).

Corte del Distrito Norte de California, 1982. Sentencia de fecha no citada, Rol No $1982-2$ Trade Cas. (CCH) P64, 774.

Corte Europea de Justicia. Sentencia de 14 de julio de 1972, Rol Caso 48/69.

Corte Europea de Justicia. Sentencia de 21 de febrero de 1973, Rol Caso C- 6/72.

Corte Europea de Justicia. Sentencia de 25 de marzo de 1999, Rol Caso T-102/96.

Corte Europea de Justicia. Sentencia de 25 de noviembre de 1971, Rol Caso 22/71.

Corte Europea de Justicia. Sentencia de 27 de septiembre de 1988, Rol casos acumulados $89 / 85$.

Corte Europea de Justicia Sentencia de 29 de junio de 2006, Rol C-301/04 p.

Corte Europea de Justicia. Sentencia de 29 de junio de 2006, Rol Caso C-289/04 P.

Corte Europea de Justicia. Sentencia de 6 de marzo de 1974, Rol Casos acumulados 6 and 7/73.

Corte Europea de Justicia. Sentencia de 6 de septiembre de 2017, Rol C-413/14 P.

Corte Europea de Justicia. Sentencia de 8 de octubre de 2008, Rol T-69/04.

Corte Europea de Justicia. Sentencia de 9 de agosto de 1994, Rol Caso C-327/91.

Corte Europea de Justicia. Sentencia de 9 de julio de 2015, Rol C-231/14 P.

Corte Europea de Justicia. Sentencia de 9 de septiembre de 2015, Rol T-91/13.

Corte Permanente de Justicia Internacional. Sentencia de 7 de septiembre de 1927 , Rol A - N ${ }^{\circ} 10$.

Corte Suprema de Estados Unidos. Sentencia de 11 de diciembre de 1922, Rol No 39.

Corte Suprema de Estados Unidos. Sentencia de 12 de junio de 1992, Rol No 91-763.

Corte Suprema de Estados Unidos. Sentencia de 14 de junio de 2004, Rol No 03-724.

Corte Suprema de Estados Unidos. Sentencia de 14 de junio de 2004, Rol No 03-724.

Corte Suprema de Estados Unidos. Sentencia de 16 de mayo de 1927, Rol No 200.

Corte Suprema de Estados Unidos. Sentencia de 19 de mayo de 1969, Rol No 49.

Corte Suprema de Estados Unidos. Sentencia de 23 de marzo de 1964, Rol No 16.

Corte Suprema de Estados Unidos. Sentencia de 24 de mayo de 1976, Rol No 73-1288425.

Corte Suprema de Estados Unidos. Sentencia de 25 de junio de 1962, Rol No 304.

Corte Suprema de Estados Unidos. Sentencia de 26 de abril de 1909, Rol No 686.

Corte Suprema de Estados Unidos. Sentencia de 26 de marzo de 1986, Rol No 83-2004.

Corte Suprema de Estados Unidos Sentencia de 28 de junio de 1993, Rol No 91-1111.

Corte Suprema de Estados Unidos. Sentencia de 29 de noviembre de 1897, Rol No 36.

Corte Suprema de Estados Unidos. Sentencia de 3 de diciembre de 1985, Rol No 84-5555.

Corte Suprema de Estados Unidos. Sentencia de 4 de junio de 1951, Rol No 352, 341.

Corte Suprema de Estados Unidos. Sentencia de 8 de enero de 1980, Rol No 78-1501. 
